\title{
Carnets
}

Revue électronique d'études françaises de l'APEF

Deuxième série - $10 \mid 2017$

Pratiques de l'errance, vécus de la mémoire

\section{L'errance, un mouvement à potentiel utopique}

Etude de trois romans de Jean Echenoz

\section{Emilie leven}

\section{(2) OpenEdition Journals}

Édition électronique

URL : http://journals.openedition.org/carnets/2265

DOI : $10.4000 /$ carnets.2265

ISSN : 1646-7698

Éditeur

APEF

Référence électronique

Emilie leven, «L'errance, un mouvement à potentiel utopique », Carnets [En ligne], Deuxième série -

10 | 2017, mis en ligne le 30 avril 2017, consulté le 30 avril 2019. URL : http://journals.openedition.org/ carnets/2265; DOI : 10.4000/carnets.2265

Ce document a été généré automatiquement le 30 avril 2019

\section{(c) (i) (8)}

Carnets est mis à disposition selon les termes de la licence Creative Commons - Atribution - Pas d'utilisation commerciale 4.0 International. 


\section{L'errance, un mouvement à potentiel utopique}

Etude de trois romans de Jean Echenoz

\section{Emilie leven}

En guise d'introduction à son livre Espèces d'espaces, Georges Perec écrit :

L'espace de notre vie n'est ni continu, ni infini, ni isotrope. Mais sait-on précisément où il se brise, où il se courbe, où il se déconnecte et où il se rassemble? On sent confusément des fissures, des hiatus, des points de friction, on a parfois la vague impression que ça se coince quelque part, ou que ça éclate, ou que ça se cogne. (...) Le problème n'est pas d'inventer l'espace, encore moins de le ré-inventer (trop de gens bien intentionnés sont là aujourd'hui pour penser notre environnement...), mais de l'interroger, ou, plus simplement encore, de le lire ; car ce que nous appelons quotidienneté n'est pas évidence mais opacité : une forme de cécité, une manière d'anesthésie (Perec, 2015 : 12).

2 Plus qu'une simple introduction ou préface, ces lignes expriment en quelques mots un questionnement fondamental et existentiel, commun à tous les êtres humains : comment être à l'espace? Quel rapport définir avec l'espace, être sédentaire ou nomade, être immobile ou en mouvement ? Ces questions sont d'autant plus percutantes qu'elles se posent avec urgence dans le contexte contemporain qui est le nôtre et qui enregistre une multiplication des mouvements migratoires, un accroissement des déplacements, une facilité toujours plus grande à se rendre d'un lieu à un autre, une démultiplication des moyens de transport et de leur fréquence, une problématisation des frontières et des identités nationales. Ces différents phénomènes, qui témoignent de l'instabilité spatiale du monde, ont un impact sur les individus qui éprouvent de plus en plus de difficultés à s'inscrire dans un lieu, à y habiter, à trouver une place dans le monde.

3 La littérature prend acte et témoigne de ces interrogations qui sont donc portées et réfléchies par les textes littéraires sur un mode qui leur est propre, celui de la fiction romanesque. A cet égard, la constatation posée par le critique littéraire $\mathrm{T}$. Ravindranathan dans son essai Là ou je ne suis pas, récits de dévoyage est particulièrement éclairante : 
A croire que l'angoisse ancienne de ne pouvoir atteindre un lieu, ou d'y être en proie à divers périls, avait fait place à celle de ne savoir l'occuper. (...) La question $\mathrm{du}$ lieu, de l'être-en-place, de l'être-dans-l'espace, maints textes clés de notre époque la lancent et relancent, comme si cette interrogation portant sur le lieu - où suis-je - pouvait tenir 'lieu' (instable, délogé, déplacé) de pensée de soi et du monde (Ravindranathan, 2012: 10 et 76).

Le rapport à l'espace contemporain, problématique et problématisé, me semble constituer un cadre pertinent au sein duquel l'étude de l'errance peut se révéler plus que pertinente. Je voudrais soutenir que l'errance, parce qu'elle me paraît être un mouvement qui questionne les usages quotidiens des routes, possède une véritable charge critique - une charge critique relevant plus précisément de l'utopie -, à même d'établir une prise de distance par rapport à nos représentations traditionnelles de l'espace et à nos modes de circulation à l'intérieur de celui-ci. L'errance serait donc un mouvement singulier qui permettrait de se réapproprier, de recréer certains rapports à l'espace, à la fois singuliers et multiples.

5 Trois grandes étapes baliseront mon propos. La première d'entre elles consistera à établir une proposition de définition de l'errance, construite à partir de l'étymologie du mot et de la philosophie de Gilles Deleuze et Félix Guattari ${ }^{1}$. Cette proposition de définition sera, dans un second temps, à confronter avec trois romans de Jean Echenoz, auteur français publié aux éditions de Minuit : Un An, Le Méridien de Greenwich, Les Grandes blondes. Enfin, il s'agira, par le biais d'une analyse qui démontre la force critique de l'errance, de mettre au jour le potentiel utopique de l'errance.

\section{Vers une définition de l'errance}

6 Selon le dictionnaire Trésor de la Langue Française informatisé (TLFi), le mot errance apparait vers la fin du XII ${ }^{\mathrm{e}}$ siècle et, par la suite, ne se manifeste que très rarement avant la moitié du XIX ${ }^{e}$ siècle. Il signifie alors "voyage, chemin » et serait un dérivé formé à partir du verbe en ancien français esrer/errer («voyager, se déplacer »), lui-même issu du verbe latin iterare signifiant «voyager ». C'est à partir de ce verbe qu'est formé le participe présent errant, attesté dès le XII ${ }^{e}$ siècle et signifiant « qui voyage sans cesse ». Cependant, comme l'attestent différents dictionnaires étymologiques ${ }^{2}$, le mot errer au sens de "voyager " possède un homonyme en ancien français : errer au sens d'« aller ça et là, s'écarter de la vérité, se tromper ", dérivé du latin errare signifiant « s'égarer, faire fausse route, se tromper ». Ces deux sens ont progressivement fusionné pour en arriver au sens général que nous connaissons aujourd'hui et dont le Larousse donne une définition claire et succincte. Selon ce dictionnaire, l'errance est «l'action d'errer », c'est-à-dire l'action d'« aller ça et là, à l'aventure, sans but » (Le Petit Larousse illustré, 2001 : 383). Le Robert, le Littré ainsi que le Trésor de la langue française témoignent de cette même fusion. On trouve dans le premier une définition quasi similaire à celle du Larousse (Le Petit Robert, 2011 : 920-921), le deuxième construit sa définition en deux points - «action de marcher, de voyager sans cesse » et « action de marcher sans but, au hasard » (Le Nouveau Littré, 2004 : 509-510) - tandis que le troisième définit l'errance comme une « situation de déplacement constant sans but " (Trésor de la langue française). En outre, ce dernier ouvrage insiste sur la dimension métaphorique du mot errer qui doit alors se comprendre au sens « d'hésiter », de « tergiverser ». 
7 L'étymologie et ces définitions de l'errance permettent de tirer plusieurs constatations. Premièrement, la courte définition de l'errance met en évidence le fait qu'il s'agit bien d'une action et ce, pour deux raisons : d'une part, le mot même action est mentionné et, d'autre part, la nature grammaticale du complément de cette action - errer, un verbe à l'infinitif - souligne cette dimension active. À l'infinitif, ces verbes manifestent l'action à l'état pur, l'action comprise comme un pur déroulement. En termes aristotéliciens, on peut dire que l'errance est une praxis, c'est-à-dire une action qui n'a pas d'autres finalités que celle de se faire, de se déployer : il s'agit bien de marcher sans but, au hasard.

Deuxièmement, la seconde partie de la définition insiste sur le fait qu'il n'y a pas de but à l'errance. L'errance est bien l'action qui fait aller çà et là, c'est-à-dire dans des endroits qui, s'ils peuvent être définis et localisables (ce qui n'est pas toujours le cas), ont cependant la particularité de toujours se trouver sur un même plan. L'expression « ça et là » illustre d'ailleurs linguistiquement cet aspect. D'une part, les lieux atteints et traversés au cours d'une errance sont comme autant de points établis sur une carte et, d'autre part, l'errance a cette capacité de lier des lieux par les trajets qu'elle dessine.

Le sens figuré de l'errance («se tromper, faire fausse route ») pointe le fait que l'errance est faite d'égarements et de détours. Puisque l'errance n'a pas de but véritable (et donc, pas de chemins ou de lieux de passage prédéfinis), l'errant ne se trompe pas réellement de destinations ou de routes, toutes peuvent être parcourues. L'errance se définit donc aussi par les détours et les chemins sinueux que l'errant parcourt. Si celui-ci s'égare de la première route qu'il avait empruntée, les pistes, sentiers et autres voies qu'il arpentera font tout autant partie de son périple.

Dans le but d'asseoir théoriquement ces considérations d'ordre étymologique et lexical, il me semble pertinent d'éclairer ces trois caractéristiques à partir de L'Anti- $\Subset$ dipe et Mille plateaux, ouvrages écrits par Gilles Deleuze et Félix Guattari. Ceci permettra de mettre au jour les trois mécanismes qui me paraissent être constitutifs de l'errance. Si l'errance est bien, comme je l'ai mentionné auparavant, une action, un mouvement, il s'agit plus précisément d'un mouvement de devenir. Suivant les deux auteurs, le devenir «ne produit pas autre chose que lui-même » (Deleuze et Guattari, 1972/1973 : 291). A l'inverse d'une ontologie essentialiste qui privilégierait les identités fixes et immuables, le devenir a ceci d'intéressant qu'il met en place une logique de mouvement intensif. Sur le corps sans organes se déploient des lignes de devenir qui ne cessent d'affecter les corps et de les faire passer par des seuils d'intensité. Les processus de devenir sont caractérisés par des lenteurs et des vitesses, au-delà - ou en-deçà - d'un mouvement effectif, localisé spatiotemporellement. C'est précisément cette variation intensive qui me parait intéressante à souligner parce qu'elle est significative de la spécificité d'un devenir fluctuant qui opère de manière libre. Dans cette perspective, errer consiste donc d'abord à se déplacer de façon libre et l'errance est un mouvement qui ne produit rien d'autre que lui-même. L'errant marche pour le mouvement en tant que tel, pour le devenir en lui-même : être errant, c'est se mouvoir dans l'espace de façon indépendante et libre, en étant affranchi de tout cadre ou but précis.

11 Si le devenir n'a pas pour visée de produire autre chose que lui-même, il faut cependant garder à l'esprit qu'il ne s'effectue pas isolément. Bien au contraire, devenir implique "une multiplicité à termes hétérogènes, et à co-fonctionnement de contagion, [qui] entrent dans certains agencements » (Deleuze et Guattari, $1980: 296)$. On ne devient pas autre mais "avec l'autre ", c'est-à-dire que le devenir établit une connexion entre une multiplicité d'éléments qui se déterritorialisent et se reterritorialisent constamment. 
Cette dernière remarque permet d'en venir au second point, à savoir la puissance connective du corps sans organes. A cet égard, les réflexions posées au début de L'AntiEdipe sont déjà révélatrices de l'importance d'une connexion libre et multiple. Contre une vision exclusive de l'inconscient et du désir, les deux penseurs privilégient le « 'soit' des combinaisons et permutations où les différences reviennent au même sans cesser d'être des différences » (Deleuze et Guattari, 1980 : 85). Sur le corps sans organes se construisent des agencements et des machines qui connectent entre eux des éléments hétérogènes selon des lignes de fuite et des devenirs singuliers. La spécificité de ces connexions est de se faire sans ordre établi (mais au contraire selon des lignes de déterritorialisation qui se renouvellent sans cesse). Cette prédominance de la connexion et de l'hétérogénéité contribue ainsi à mettre sur pied un modèle rhizomatique de la pensée. Le rhizome, terme qui tire son origine de la biologie, se présente sous la forme d'une racine à multiples embouts, qui se développe selon des configurations multiples: «Un rhizome peut être rompu, brisé en un endroit quelconque, il reprend suivant telle ou telle de ses lignes et suivant d'autres lignes » (Deleuze et Guattari, 1980 : 16). Dans cette perspective, l'errance se comprend comme un mouvement qui trace des lignes entre des points situés sur une même surface. Les trajets de l'errant connectent villages, villes et pays et ceux-ci sont entièrement subordonnés à ceux-là.

12 En outre, les lignes du rhizome ont ceci de particulier qu'elles « oscillent entre les lignes d'arbres qui les segmentarisent et même les stratifient, et des lignes de fuite ou de rupture qui les emportent » (Deleuze et Guattari, 1980 : 632). En d'autres termes, on ne peut opposer territorialisation et déterritorialisation ${ }^{3}$ sans penser la tension et le lien qui existent entre les deux pôles. Cette dernière observation mène au dernier point d'analyse. Comme je l'ai écrit dans l'esquisse de définition, l'errance semble faite de détours et de déviations. Ces détours peuvent se comprendre comme les oscillations qui s'établissent sans cesse entre organisme et corps sans organes, entre points et lignes, entre espace strié et espace lisse, entre lignes molaires et lignes moléculaires, entre sédentarisme et nomadisme, entre territoires et lignes de fuite. Ces différents couples d'opposés ne sont pas saisis dans une logique binaire d'exclusion. Bien au contraire, l'échange est permanent :

Entre les deux pôles, il y a tout un domaine de négociation, de traduction, de transduction proprement moléculaire, où tantôt les lignes molaires sont déjà travaillées par des fissures et des fêlures, tantôt les lignes de fuite, déjà attirées vers des trous noirs, les connexions de flux, déjà remplacées par des conjonctions limitatives (Deleuze et Guattari, 1980 : 273).

13 Ces quelques lignes illustrent très clairement l'entrelacement dynamique et nécessaire qui lie chaque série des termes repris ci-dessus. En ce sens, errer se présente comme un mouvement qui ne cesse d'osciller entre déterritorialisation et territorialisation. Pour le dire autrement, celui qui erre dessine de nouveaux trajets de façon libre, mais il n'est jamais à l'abri de retomber sous le joug d'un pouvoir extérieur qui déciderait alors de ses mouvements et de ses déplacements.

Sur la base de ces considérations d'ordre étymologique, lexical et théorique, je proposerai une ébauche de définition de l'errance centrée autour de trois points qui spécifient les trois mécanismes constitutifs de cette manière d'être à l'espace : l'errance se présente comme un mouvement de devenir libre doué d'une capacité de connexion et toujours susceptible d'être reterritorialisé, c'est-à-dire ramené dans des itinéraires définis et assujetti à un but extérieur. Ces trois mécanismes seront les trois angles d'approche à 
partir desquels j'aborderai l'analyse de trois romans de Jean Echenoz : Un An, Le Méridien de Greenwich, Les Grandes blondes.

\section{Cartographie de trois mouvements d'errance}

15 Avant d'entamer l'étude des mouvements d'errance dans ces trois romans, quelques mots sur leur diégèse respective. Un An met en récit l'errance de Victoire qui, pendant une longue année, passe d'un lieu à l'autre sans itinéraire prédéfini. Constatant la mort de Félix ${ }^{4}$, son compagnon du moment, et voulant éviter les soupçons et les accusations, la jeune femme décide de partir sur les routes et embarque dans le premier train qui se présente à elle. Pendant une année, elle erre dans le Nord de la France, habite temporairement maisons louées, chambres d'hôtels et abris de fortune. Au terme d'un an de déplacements, Victoire retourne à Paris et retrouve son ancienne vie. Le Méridien de Greenwich narre la longue aventure de deux sociétés secrètes qui cherchent à retrouver Byron Caine, un étrange scientifique. Celui-ci, en charge d'un projet de haute importance, s'est enfui sur une île et y continue son travail. Tout au long du roman, les tenants et aboutissants de l'histoire restent flous : les personnages et les lecteurs demeurent dans une certaine confusion par rapport aux événements qui surviennent. Après plusieurs retournements de situation, le roman se conclut singulièrement : l'île explose, le projet disparait, la plupart des personnages meurent et les seuls survivants partent pour le pôle Nord. Le roman Les Grandes blondes, quant à lui, raconte la course-poursuite entre d'apparents détectives privés et Gloire Abgrall, une ancienne star du cinéma. Cette dernière, recherchée par une société de télévision, a disparu depuis quatre ans et tient à garder l'anonymat. Aidée de Béliard, personnage fantastique faisant office d'angegardien, la jeune femme traverse les continents afin d'échapper à ses poursuivants.

Trois romans, trois errances, à la fois singulières et similaires. Ces mouvements sont singuliers car ils se déploient chacun dans un cadre spécifique et occupent plus ou moins de place au sein des romans : ainsi, l'errance est prépondérante au sein d'Un An, apparaît ponctuellement dans Le Méridien de Greenwich et occupe une position intermédiaire au sein des Grandes blondes). Cependant, ces errances se rapprochent et se solidarisent dans la mesure où elles permettent de faire émerger des enjeux communs et sont analysables à partir d'un même angle d'approche, un angle d'approche à trois entrées.

17 La première d'entre elles, comme je l'ai mentionné plus haut, est celle qui conçoit l'errance comme un mouvement de devenir. Ainsi, dans les trois cas, ce qui importe réellement n'est pas l'endroit ni le but en eux-mêmes mais bien le fait d'être mobile. Dans Un An, Victoire ne cesse de se déplacer d'un lieu à l'autre. Chaque nouveau lieu et chaque direction sont choisis au hasard, comme l'illustre clairement le passage suivant, situé à peu près à la moitié du roman :

Faute de se résoudre encore à rejoindre une grande ville, elle continuait de choisir au hasard sur sa carte, souvent sur la foi du seul son de leur nom, des agglomérations mineures où elle tâchait toujours de se nourrir et s'abriter pour une ou deux nuits. Cela produirait une errance en dents de scie, pas très contrôlée : s'il se pourrait qu'on fit quelque détour pour l'avancer, il arriverait aussi qu'elle dût s'adapter à une destination, ceci équilibrant cela. Son itinéraire ne présenterait ainsi guère de cohérence, s'apparentant plutôt au trajet brisé d'une mouche enclose dans une chambre (Echenoz, $1997: 63$ ).

Cette errance en dents de scie commence directement, dès le début du roman. Ainsi, Victoire choisit sa première destination sans y réfléchir, motivée uniquement par le désir 
de partir et de s'éloigner au plus vite : "Victoire chercha sur un écran le premier train capable de l'emmener au plus vite et le plus loin possible: l'un, qui partait dans huit minutes, desservirait Bordeaux » (Echenoz, 1997 : 8). Une fois à Bordeaux, elle embarque dans un nouveau train qu'elle choisit au hasard, pour « brouiller les pistes » et poursuivre son périple. La suite du roman se construit selon une même logique : Victoire se déplace en « direction de n'importe où » et s'oriente « n'importe comment, au gré des panneaux indicateurs et sans but précis " (Echenoz, 1997: 81 et 96). Le roman insiste sur le caractère secondaire des destinations au profit du mouvement qui permet à Victoire de passer d'un lieu à l'autre.

Dans Le Méridien de Greenwich, un personnage attire plus particulièrement l'attention : il s'agit de Théo Selmer, traducteur employé par l'ONU. Du jour au lendemain, ce personnage quitte son travail, traverse l'Amérique latine où il commet un triple meurtre et devient membre de l'organisation secrète dirigée par Carrier. Alors que Théo Selmer vit une existence relativement simple et normale, celui-ci modifie radicalement son comportement et tombe alors dans une forme d'errance très particulière. Ce changement frappe non seulement par son aspect soudain mais aussi par son absence totale de raison précise. Tandis que le personnage se rend à son travail comme à son habitude, les choses changent soudainement: "Puis, sans qu'il sût très bien comment ni pourquoi, brusquement, il cessa ; arrêta de traduire, de travailler, de fonctionner ; se mit en grève " (Echenoz, 1979: 63). Suite à ce brusque arrêt, l'homme semble alors agir uniquement en fonction de ses pulsions. Théo Selmer consacre les mois suivants « à se mouvoir en zigzag à travers l'Amérique du Sud " (Echenoz, 1979: 64) et ce, sans s'arrêter : "Saisi par une sorte d'impatience perpétuelle, il lui devint impossible de demeurer plus de quinze jours dans une même ville » (Echenoz, 1979: 65).

Enfin, personnage principal du roman Les Grandes blondes, Gloire Abgrall est une femme disparue depuis plusieurs années. Après un bref succès à la télévision et une déchéance aussi rapide que soudaine, « le parcours perceptible de Gloire Abgrall s'interrompt net le jour de sa sortie de prison» (Echenoz, 2006 : 31-32). Depuis quatre ans, la jeune femme se cache dans un petit village de Bretagne et «a coupé toute relation passée, une deuxième fois elle a changé de nom, se faisant appeler Christine Fabrègue, et transformé son apparence » (Echenoz, 2006: 69). Cependant, des hommes engagés par une société de télévision finissent par retrouver l'ancienne star. Celle-ci quitte alors sa maison, s'échappe et débute un voyage imprévisible. Au cours de son parcours d'errance, la jeune femme arpente le monde et traverse de nombreuses villes telles que Paris, Sydney et Bombay. Ce mouvement d'errance, également mouvement de fuite, frappe par son imprévisibilité et sa radicalité. La violente réaction de Gloire suite aux visites de ses poursuivants et sa prompte décision à s'en aller témoignent de ces deux aspects.

Routes empruntées et villes traversées importent donc peu, l'essentiel étant d'être mobile. Ni Victoire, Ni Théo Selmer, ni Gloire Abgrall ne voyagent pour visiter ou pour rejoindre des amis, ils ne planifient ni leurs prochaines destinations, ni leurs prochaines activités. Rien n'est prévisible, l'essentiel consiste à enchainer les déplacements pour se maintenir en mouvement. De ce point de vue, les errances des trois personnages se présentent comme des mouvements de devenir, des mouvements libres et n'ayant aucun but si ce n'est celui de se déployer.

Par ailleurs, dans les trois romans, les descriptions des trajets et déplacements l'emportent toujours sur celles des destinations. Un An est le roman le plus emblématique de cet aspect car, tandis que les indications relatives aux lieux se font de plus en plus 
maigres au fil du récit, celles concernant les trajets et moyens de transport prennent de plus en plus d'importance. Tout en primant sur les lieux, le mouvement assure la connexion entre chacun d'entre eux. Les différents points qui constituent l'itinéraire des personnages s'agencent grâce à leurs déplacements et sont dotés d'un statut analogue : il s'agit de lieux dans lesquels les personnages s'arrêtent temporairement sans jamais les investir personnellement. Qui plus est, ces transports et ces lieux sont placés sous le signe commun du mouvement, d'« un monde (...) promis à l'individualité solitaire, au passage, au provisoire et à l'éphémère » (Augé, 1992: 100). En effet, qu'il s'agisse d'hôtels, de places publiques, d'abris, de trains ou de cars, les romans regorgent de ce qu'on peut appeler, à la suite de Marc Augé, des non-lieux. L'auteur, qui forge ce terme dans un essai anthropologique intitulé Non-lieux: introduction à une anthropologie de la surmodernité, cite comme non-lieux les espaces suivants, espaces mobiles ou immobiles :

Voies aériennes, ferroviaires, autoroutières et les habitacles mobiles dits 'moyens de transports' (avions, trains, cars), les aéroports, les gares et les stations aérospatiales, les grandes chaînes hôtelières, les parcs de loisir, et les grandes surfaces de la distribution, l'écheveau complexe, enfin, des réseaux câblés ou sans fil (Augé, 1992 : 101-102).

Enfin, on observe dans les trois romans un mécanisme de territorialisation qui vient nuancer la dimension libre et spontanée de l'errance des personnages. Dans Un An, cette territorialisation s'actualise par l'intermédiaire de Louis-Philippe, ami de Victoire et personnage énigmatique dont la particularité réside dans sa capacité à toujours retrouver la jeune femme. Installée depuis peu dans le pavillon de Saint-Jean-de-Luz, Victoire reçoit la visite inattendue de Louis-Philippe :

Retenant le battant, Victoire se pencha silencieusement vers l'extérieur sans reconnaître aussitôt l'intrus qui, tête par avance renversée en arrière, regardait dans sa direction. Mais qu'est-ce que tu fais-là ? dit-elle. Ouvre-moi, répondit LouisPhilippe (Echenoz, 1997 : 29-30).

Cette faculté à retrouver la jeune femme est dotée d'un effet reterritorialisant ${ }^{5}$ : la jeune femme ne se libère jamais entièrement de ce qu'elle fuit. Cet aspect est d'autant plus marquant que Louis-Philippe donne systématiquement à Victoire des nouvelles de Félix. Par ailleurs, c'est une rencontre avec Louis-Philippe qui mettra fin à l'errance de Victoire. Dans le débit d'autoroute qui abrite leur dernière rencontre, la conversation se porte à nouveau sur Félix :

L'affaire Félix était close, fit-il savoir, il ne fallait plus y penser. On avait fini par la classer en écartant toute responsabilité de Victoire. Bien que sa disparition eût d'abord intrigué, on n'avait qu'à peine envisagé de retenir quoi que ce fût contre elle. Pas de soupçon ni même de supposition : elle pouvait maintenant rentrer à Paris (Echenoz, 1997 : 104).

De fait, après cette conversation, Victoire reprend sa route et se rend en auto-stop jusque Paris. Ces deux rencontres se situent respectivement au début et à la fin de l'errance de Victoire, qui se révèle donc encadrée par les interventions de Louis-Philippe.

Dans Le Méridien de Greenwich, la dimension impulsive et libre de l'errance de Théo Selmer est contredite dans la suite du roman. En effet, après ses pérégrinations en Amérique latine, Selmer rentre à Paris et rencontre Carrier. Au fil de leur conversation, Carrier fait peu à peu comprendre au traducteur que celui-ci travaillait déjà pour son organisation mais sans en être conscient. Un extrait de leur dialogue permet d'éclairer ces derniers propos : 
- Je ne travaillais même pas, argumenta-t-il. Je ne travaillais quand même pas pour vous?

- Bien sûr que si, dit Carrier.

(...)

Eh bien voilà, fit Carrier avec une sorte de gêne, comme s'il tentait d'expliquer un théorème à un trisomique. Voilà, c'est très simple. A partir de là, nous vous avons observé quelques temps. Et puis, quand nous avons eu l'impression que vous réagiriez comme il était souhaitable, il a suffi de faire en sorte que les Américains prennent le même autocar que vous.

(...)

C'était délicat. Il était impératif que personne ne puisse remonter à nous à partir de cette histoire. Si vous aviez échoué, si vous vous étiez fait prendre, personne n'aurait pu savoir que c'était nous qui avions monté l'affaire, puisque vous-même ne le saviez pas (Echenoz, 1979 : 100-101).

Ces quelques lignes témoignent d'un retournement complet de situation. Ce bref passage démontre que l'errance de Selmer, loin d'être libre et pulsionnelle, est en réalité soumise à un pouvoir extérieur. Sous l'apparence d'une errance libre et autonome, le mouvement de Théo Selmer se révèle être le fruit d'une organisation externe.

Enfin, dans Les Grandes blondes, la liberté de décision de Gloire se perd subitement et les déplacements du personnage sont alors le résultat de décisions externes et indépendantes de sa propre volonté. Après quelques voyages, Gloire rencontre des trafiquants qui la persuadent et l'obligent à travailler pour eux. A deux reprises, la jeune femme se voit confier une mission qu'elle ne peut pas refuser :

J'aimerais vous confier un objet que je veux faire parvenir à mon parent de Bombay.

(...) Ça vous dit de vous en occuper? Voyage payé, bien sûr (Echenoz, $2006: 161$ ).

C'est tout simple, dit Moopanar, je dois expédier quelque chose dans votre pays. Il

s'agirait seulement d'accompagner cette chose. Veiller à ce que tout se passe bien.

Etre là, quoi (Echenoz, 2006 : 185-186).

Dans ces deux exemples, on observe une entière reterritorialisation des mouvements de Gloire. Au départ libre et relativement autonome, l'errance de la jeune femme se retrouve subordonnée à un pouvoir extérieur, c'est-à-dire la volonté des trafiquants. Nous observons donc un changement progressif et complet des mouvements de Gloire qui, d'instinctifs à libres, deviennent soumis et contraints. D'une manière générale, Les mouvements d'errance et de reterritorialisation ne sont donc pas opposés l'un à l'autre dans des positions statiques mais reliés par une ambivalence dynamique.

\section{L'errance, un mouvement à potentiel utopique ?}

Sur la base de ces analyses, je voudrais montrer dans quelle mesure ces trois mouvements d'errance sont dotés d'un potentiel critique et, plus précisément, utopiquement critique. Ce potentiel de l'errance - qui réside selon moi dans la capacité qu'a ce mouvement à remettre en question les manières de se rapporter à l'espace - n'est pensable que dans la mesure où l'on explicite le lien fondamental entre espace et utopie. En effet, l'utopie, qu'il s'agit ici de penser non plus en tant que société idéale, mais en tant que potentiel ou puissance de rupture et d'interrogation, me semble avoir comme condition de possibilité l'ambigüité spatiale qui la constitue et qu'elle travaille.

Qu'il s'agisse de Paul Ricœur, de Pierre Macherey ou de Miguel Abensour, les différents philosophes qui ont réfléchi au concept d'utopie au cours du $\mathrm{xx}^{\mathrm{e}}$ siècle mettent tous en évidence la puissance critique dont elle est dotée. Par ailleurs, si l'utopie peut se 
comprendre comme mise à distance du réel et remise en cause de l'ordre établi, elle est également mise à distance de sa propre effectivité : l'utopie n'a pas pour but de se réaliser. D'ordre fictionnelle, l'utopie opère un court-circuitage entre le contexte socioculturel à partir duquel elle émerge et les espaces spatiaux-temporels, lieux de nulle part, qu'elle met en récit; elle ne vise jamais sa réalisation effective. Ce paradoxe, de nature logique, est important à mentionner car il me semble prendre source dans un paradoxe spatial, un paradoxe qui ferait justement jouer les catégories de l'espace, le «là » et le " pas là », le « lieu » et le «non-lieu », l'inscription dans le monde et l'insularité radicale de l'utopie, ceci indiquant à nouveau le lien fondamental et étroit qui relie espace et utopie. Celle-ci est en effet intéressante à réfléchir en tant qu'espace singulier qui est non-lieu, c'est-à-dire en tant que lieu niant son inscription dans l'espace tout en affirmant ne pas être sans rapport avec celui-ci. Cette dimension de non-lieu fait droit à la négativité qui constitue l'utopie et c'est précisément cette négativité qui permettrait de faire jouer l'utopie comme double mise à distance. En effet, la dimension de non-lieu est tout d'abord un moyen d'éviter toute délimitation définitive de l'utopie comme espace figé et ne cesse de mettre en mouvement l'effectivité des cadres spatio-temporels du présent. Ensuite elle empêche toute réalisation effective de l'utopie car celle-ci supposerait que le non-lieu devienne entièrement lieu et, par-là, l'utopie se dénaturerait entièrement pour se figer en une réalisation spatiale donnée, démise de toute puissance critique.

Pour en revenir aux trois romans d'Echenoz, l'errance des personnages me semble dotée d'un potentiel utopique dans la mesure où elle permet à ceux-ci d'échapper à une situation problématique et met alors en place une manière singulière d'être dans l'espace. Dans les trois cas, partir apparaît comme la seule solution possible pour les personnages. Ainsi, face aux problèmes qui se posent à elles, Victoire et Gloire réagissent immédiatement et similairement : il faut quitter les lieux et s'en aller. L'incipit d'Un An est particulièrement significatif à cet égard puisqu'en une phrase sont posés le problème et le départ du personnage : « Victoire, s'éveillant un matin de février sans rien se rappeler de la soirée puis découvrant Félix mort près d'elle dans leur lit, fit sa valise avant de passer à la banque et de prendre un taxi vers la gare Montparnasse » (Echenoz, 1997: 8). Au sein du Méridien de Greenwich, partir est l'unique moyen que trouve Théo Selmer pour se tirer de la situation d'engluement et d'ennui dans laquelle il est pris au piège.

Cette mise en mouvement est l'amorce du potentiel utopique de l'errance. Outre le fait que le début de l'errance des personnages débloque ceux-ci de la situation dans laquelle ils étaient englués, ceux-ci se réappropriant alors une certaine forme de liberté, leur mouvement d'errance leur permet de créer un nouveau rapport à l'espace, à la fois critique et singulier. Tout d'abord, de par son imprévisibilité et parce qu'elle n'a aucun but si ce n'est celui d'être un mouvement qui se déploie pour lui-même, l'errance détourne l'usage quotidien des routes: habituellement moyens, intermédiaires par le biais desquels voyageurs et usagers des routes réalisent un but précis (par exemple, arriver à une destination), les routes non seulement deviennent pour les personnages la condition de leur liberté mais acquièrent, de façon plus générale, une autonomie propre : les routes valent en tant que routes, en tant qu'espace qui est condition de possibilité et actualisation de l'être en errance.

34 En outre, l'imprévisibilité de l'errance permet aux personnages d'échapper aux logiques de quadrillage et de rationalisation des déplacements, logiques structurantes au sein du monde contemporain. Sans aucun but et spontanée, l'errance apparaît comme une façon 
d'être à l'espace qui remet en question le répertoriage systématique des déplacements et la localisation permanente de tout un chacun (l'exemple du GPS n'en n'est qu'un parmi d'autres), la tendance de plus en plus accrue à se mouvoir le plus efficacement possible dans un but précis et ce, qu'il s'agisse d'un but économique, politique ou encore récréatif.

Ensuite, l'errance permet la remise en question d'un mode d'être à l'espace stationnaire, fixe, et ce, au profit d'un mode qu'on pourrait appeler en transit permanent. Dans les trois cas qui nous occupent, les personnages quittent leur environnement quotidien pour une vie d'errance qui deviendra leur nouvelle manière d'être à l'espace. A l'inverse d'une vision qui privilégierait la sédentarité, l'errance des trois personnages - qui ne cessent de passer d'un lieu à l'autre, de prendre une multitude de transports - fait du mouvement le principe opérant de leur manière d'être au monde. De ce fait, c'est aussi et plus particulièrement la manière d'habiter un lieu qui est questionnée. Qu'il s'agisse de Théo Selmer et Gloire Abgrall qui n'occupent que temporairement des chambres d'hôtels ou de Victoire qui multiplie les façons d'habiter (appartement provisoire d'abord, hôtels ensuite, abris et vie en autogestion enfin), il y a dans les trois romans une remise en question de l'habitation permanente et de la propriété privée au profit d'une manière d'habiter plus souple et plus mobile.

Enfin, il me semble que les connexions permises par l'errance, qui met sur un même plan les différents lieux par lesquels passent les personnages (capitales, grandes villes mais aussi petits villages), permet une remise en cause de la hiérarchie entre centre et périphérie. A l'inverse d'une vision centriste mettant sur piédestal une capitale par rapport à laquelle se rapportent tous les autres lieux, ici est privilégiée une vision que l'on pourrait qualifiée de rhizomatique. Celle-ci nous invite à repenser les liens et les rapports de domination entre grosses villes et petits villages, au profit d'une vision spatiale où chaque lieu occupe une place d'importance équivalente.

Notion riche et complexe s'il en est, l'errance me semble déployer un potentiel utopique fort, permettant d'interroger et de remettre en question nos rapports à l'espace. Ce potentiel d'utopique, dont j'ai tenté de montrer dans cet article les actualisations au sein de trois romans de Jean Echenoz, me semble d'autant plus intéressant à analyser que la question de l'espace - et sa problématisation - se pose avec de plus en plus d'insistance dans la littérature contemporaine.

\section{BIBLIOGRAPHIE}

ABENSOUR, Miguel (2000). L'utopie de Thomas More à Walter Benjamin. Paris : Sens \& Tonka. ANALYSE ET TRAITEMENT INFORMATIQUE DE LA LANGUE FRANÇAISE. «Trésor de la Langue Française informatisé (TLFi) » [en ligne]. France : CNRS \& Université de Lorraine [disponible le 18/06/2016]< http://atilf.atilf.fr/tlf.htm>.

AUGÉ, Marc (1992). Non-lieux. Introduction à une anthropologie de la surmodernité. Paris : Seuil, « La librairie du xxe siècle ». 
BAUMGARTNER, Emmanuèle \& MÉNARD, Philippe (1996). Dictionnaire étymologique et historique de la langue française. Paris : Librairie générale française, « Le Livre de Poche».

BLUM, Claude \& PRUVOST, Jean \& ALAOUI, Khalid (e.a) (2004). Le Nouveau Littré (éd. augmentée).

Varese : Garnier.

DELEUZE, Gilles \& GUATTARI, Félix (1972/1973). L'Anti-@Edipe. Capitalisme et schizophrénie 1. Paris :

Minuit, «Critique ».

Deleuze, Gilles \& GUATTARI, Félix (1980). Mille Plateaux. Capitalisme et schizophrénie 2. Paris : Minuit, «Critique».

ECHENOZ, Jean (1979). Le Méridien de Greenwich. Paris : Minuit.

ECHENOZ, Jean (1997). Un An. Paris : Minuit.

ECHENOZ, Jean (2006). Les Grandes Blondes. Paris : Minuit, « Double, 34 ».

LAROUSSE (2001). Le Petit Larousse illustré. Paris : Larousse.

MACHEREY, Pierre (2011). De l'utopie! Paris : De l'Incidence Editeur.

MITTERAND, Henri, DUBOIS, Jean, DAUZAT, Albert (1994). Dictionnaire étymologique et historique du français. Paris : Larousse.

PEREC, Georges (2000). Espèces d'espaces. Paris : Galilée, « L'espace critique».

PICOCHE, Jacqueline (1971). Nouveau dictionnaire étymologique du français. Paris : Hachette-Tchou, «Les usuels».

RAVindranATHAN, Thangam (2002). Là Où Je ne suis pas. Récits de dévoyages. Paris : Presses

Universitaires de Vincennes, «L'imaginaire du texte».

RICœUR, Paul (2005). L'idéologie et l'utopie. Paris : Seuil.

ROBERT (2011). Le Petit Robert. Paris : Le Robert.

\section{NOTES}

1. Je mobiliserai ici les réflexions menées par Deleuze et Guattari dans leur diptyque Capitalisme et schizophrénie. Je résume très brièvement les grandes lignes de leur démarche. Dans un premier temps (celui de L'Anti-ÆEdipe, que l'on peut considérer comme le versant critique de leur entreprise), les deux auteurs mettent en procès les modes d'aliénation à l'œuvre dans le capitalisme et dans la psychanalyse œdipienne et ce, en confrontant leur régime respectif. Ils s'attèlent à penser l'inconscient comme une machine désirante et à montrer que le désir ne cesse d'investir les formations sociales. Dans un second temps (celui de Mille plateaux, considéré comme le pendant créatif et positif de L'Anti-Oedipe), les deux penseurs établissent une série de réflexions qui font justement droit au désir, montrent sa puissance de production, sa capacité à déterritorialiser les structures fixes et à provoquer des lignes de fuite créatrices.

2. Cf. BAUMGARTNER, Emmanuèle \& MÉNARD, Philippe (1996). Dictionnaire étymologique et historique de la langue française. Paris: Librairie générale française (Le Livre de Poche), p. 292 ; MITTERAND, Henri \& DUBoIS, Jean \& DAUZAT, Albert (1994). Dictionnaire étymologique et historique du français. Paris : Larousse, pp. 264-265; PICOCHE, Jacqueline (1971). Nouveau dictionnaire étymologique du français. Paris : Hachette-Tchou (Les usuels), p. 255. 
3. Pour éviter un flou conceptuel, territorialisation signifie plus précisément rentrer dans le droit chemin, dans le système social ou la structure de l'ordre établi, alors que déterritorialisation signifie en sortir, prendre un chemin de traverse.

4. En réalité, Félix n'est pas véritablement mort. Son cœur s'est arrêté pendant quelques instants, pour ensuite se remettre à battre. La fin du roman et la lecture de Je m'en vais permettent de comprendre ces éléments.

5. C'est-à-dire d'un effet qui ramène Victoire vers ce à quoi elle tentait d'échapper.

\section{RÉSUMÉS}

Cet article se propose d'analyser le potentiel utopique de l'errance et de réfléchir à celle-ci en tant que mouvement permettant de se réapproprier, de récréer certains rapports à l'espace, à la fois singuliers et multiples. Sur la base d'une tentative de définition de l'errance (construite à partir de l'étymologie du mot ainsi que de la philosophie de Deleuze et Guattari - plus précisément, les ouvrages L'Anti-(Edipe et Mille plateaux) et d'une analyse de trois romans de Jean Echenoz (Un An, Le Méridien de Greenwich, Les Grandes blondes), cet article cherchera donc à montrer que l'errance possède une véritable charge utopique, à même de permettre au personnage contemporain de nouer des rapport singuliers avec l'espace, en déjouant et en répondant aux problèmes et questions que celui-ci pose.

This paper aims to enlighten the utopian power of wandering which creates new connections and relations with and inside space. For this purpose, this analysis will defend a new definition of the term of wandering itself, based on the etymology of the word as well as on the philosophy of Deleuze and Guattari. By focusing on three of Jean Echenoz's novels (Un An, Le Méridien de Greenwich, Les Grandes blondes), this paper will finally demonstrate the specific utopian power of wandering which confers to the contemporary character new ways to elaborate and respond to spatial issues.

\section{INDEX}

Mots-clés : errance, potentiel utopique, espace, Echenoz (Jean)

Keywords : wandering, utopian power, space, Echenoz (Jean)

\section{AUTEUR}

\section{EMILIE IEVEN}

Université Saint-Louis - Bruxelles

emilie.ieven[at]usaintlouis.be 